\title{
Germanica
}

\section{Gobi de Tor Åge Bringsværd. Histoire(s), Histoire, Historia : de la fascination de raconter}

Gobi de Tor Åge Bringsvoerd. Geschichte(n), Historia : faszinierendes Erzählen

Régis Boyer

\section{CpenEdition}

\section{Journals}

Édition électronique

URL : http://journals.openedition.org/germanica/1258

DOI : $10.4000 /$ germanica. 1258

ISSN : 2107-0784

Éditeur

Université de Lille

\section{Édition imprimée}

Date de publication : 1 décembre 1998

Pagination : 77-91

ISBN : 0984-2632F

ISSN : 0984-2632

Référence électronique

Régis Boyer, «Gobi de Tor Åge Bringsværd. Histoire(s), Histoire, Historia : de la fascination de

raconter », Germanica [En ligne], 23 | 1998, mis en ligne le 26 janvier 2012, consulté le 06 octobre 2020.

URL : http://journals.openedition.org/germanica/1258; DOI : https://doi.org/10.4000/germanica. 1258

Ce document a été généré automatiquement le 6 octobre 2020.

(c) Tous droits réservés 


\title{
Gobi de Tor Åge Bringsværd. Histoire(s), Histoire, Historia : de la fascination de raconter
}

\author{
Gobi de Tor Åge Bringsvoerd. Geschichte(n), Historia : faszinierendes Erzählen
}

\author{
Régis Boyer
}

1 Permettez-moi d'ouvrir ce petit essai par deux réflexions, accordées l'une à l'autre, au demeurant.

2 La première est qu'un bon Scandinave est premièrement un narrateur, un conteur, un raconteur. Statistiquement, si l'on ose dire, car cet adverbe est toujours d'une manipulation dangereuse, le Nord a écrit beaucoup plus de prose narrative que de poésie ou de théâtre, sans parler d'essais. Si la liste de ses grands auteurs compte des noms de tout premier ordre comme L. Holberg, A. Strindberg ou H. Ibsen (qui ne furent pas que des dramaturges, tant s'en faut), si, Dieu merci, les poètes de haut vol ne manquent pas, les romanciers, auteurs de nouvelles, conteurs, tiennent, et de fort loin, le haut du pavé. Après tout, Andersen est Danois, Selma Lagerlöf, Suédoise, et Knut Hamsun, Norvégien. Quant aux sagas, elles sont à peu près exclusivement islandaises. Comme si l'inspiration Scandinave tendait à se résoudre, de préférence, avec une nette alacrité, en prose narrative. Et j'y ajoute tout de suite une précision capitale pour la présente réflexion : dans leur immense majorité, ces nouvelles, ces romans relèvent à des degrés divers du genre dit historique. Je veux dire par là que toute la gamme imaginable d'attitudes en face du donné de l'Histoire se rencontre avec une profusion remarquable, de la simple incitation à l'application à coïncider, si faire se peut, avec ce que put être la "réalité ». Je me suis toujours demandé pourquoi, même dans ce domaine que privilégie le présent ouvrage, la fantaisie débridée, la fiction pure, l'analyse psychologique en soi, l'étude attentive des élans du cœur, l'autobiographie romancée destinée à illustrer un cas d'espèce, la réflexion philosophique traduite par des arguments et des personnages fabriqués à cette fin, l'application à dominer une certaine conception de la temporalité, etc. sont finalement si peu au rendez-vous. Tant elles sont écrasées soit par l'Histoire au sens «scientifique» du terme, ou par des 
mythes (Don Juan par exemple), des images (tout l'arrière-plan conventionnel de l'aventure viking), des légendes (Ahasvérus dont il est surprenant de voir le succès qu'il a connu dans le Nord, de Kierkegaard à Lagerkvist) et ainsi de suite. Gratuité, démesure, irréalité sont très rarement au rendez-vous de ces inspirations.

En second lieu, je ne suis pas sûr d'avoir raison lorsque je dis que ce prétexte (puisqu'en somme, tel est bien le sens de ce que je propose ici) historique est indispensable et, donc, premier. Je parlais de narration, de conte : j'ai toujours été frappé du fait que, même dans la vie courante, au hasard des conversations, un bon Scandinave est premièrement un homme qui raconte. Qui illustre ses pensées ou ses sentiments par une anecdote bien troussée, une réminiscence vivante si possible, l'évocation d'un fait plus ou moins vécu. Et c'est également l'impression que j'emporte presque à coup sûr en lisant quelque grand texte en prose du Nord: voilà l'auteur parti, il tient son argument, il entreprend de le développer. Il se peut qu'il suive un fil chronologique rigoureux ou qu'il soit fidèle à un modèle qu'il s'est donné ; il arrive que l'idée-force qui le mène (je pense à Johannes $\mathrm{V}$. Jensen ou à Sigrid Undset) dicte la progression de son œuvre, serait-elle considérable; ou qu'un temps au moins, une idéologie, quelle qu'elle soit, entende s'incarner dans les personnages que l'on nous donne à voir et leurs heurs et malheurs. Mais, sauf exceptions, cela ne dure qu'un temps. A un moment donné, la verve, le bonheur d'écrire, la passion de raconter qui sont la marque propre de tout écrivain Scandinave l'emportent sur le possible argument, et le voilà parti. Digression ici, long développement adventice là, écarts apparemment incongrus dans toutes sortes de domaines où l'Histoire avec majuscule se perd (je songe aux grands romans romantiques flamboyants, et prétendument historiques, de CJ.L. Almquist) et nous sommes pris, il n'y a aucun moyen de résistance, nous n'échapperons pas à cette étrange fascination.

C'est un aspect que j'ai beaucoup étudié chez Knut Hamsun. Comme on le sait, une partie non négligeable de son œuvre part d'un prétexte plus ou moins historique, lorsqu'il fait la satire de la société capitaliste et de ses prétendus idéaux qu'il oppose aux vertus saines et primitives de la vie à l'ancienne. Et je consens sans peine qu'il y a une satire souvent féroce de nos idéaux modernes, sociaux aussi bien qu'économiques, moraux comme religieux, derrière de vastes fresques comme le diptyque Enfants de leur temps - La ville de Segelfoss.Cela, toutefois, ne nous est pas dit avec la clarté ou la brutalité qui viennent de dicter la phrase précédente : cela nous est communiqué, à l'indirecte, à la Knut Hamsun, par son fameux personnage de vagabond-qui-n'acceptepas-la-condition-humaine-telle-qu'on-nous-1'inflige. Et ce qu'il a de prodigieux, ce Knut Pedersen ou Johan Nagel ou Tomas Glahn ou August (August surtout, en vérité, qui pèse du même poids fondamental que le «je » de Faim), c'est qu'il parle, parle, raconte, raconte. Paraphrasons un bref passage d'August.L'intarissable hâbleur, phraseur, vantard est reparti dans l'une de ses inénarrables histoires et ses auditeurs ne peuvent s'empêcher de s'écrier (en substance) : «Parle! Parle encore, August! Nous savons bien que tu mens\{c'est moi qui souligne\}, mais tu parles comme un journal, parle, parle encore! » Je ne m'interrogerai pas ici sur les possibles justifications de pareilles attitudes, il est clair qu'elles peuvent fort bien ressortir à des vérités d'ordre social ou psychologique (la fameuse « timidité », à laquelle je ne crois pas, au demeurant, qui, par compensation, amènerait une pareille prolixité - mais pourquoi, alors, dans le genre narratif et appliqué à des arguments plutôt historiques ?), mais mon propos n'est pas de saisir l'occasion pour faire de l'ethno-psychologie. Je redis simplement que l'une des raisons de l'évidente fascination qu'exercent sur nous les littératures Scandinaves tient 
à leur pouvoir souverain de raconter des histoires, ou à faire de l'Histoire, romancée ou non. En d'autres termes, que, derrière tout bon romancier du Nord, il y a en dernière analyse une voix, un ton, des accents tout à fait caractéristiques, et que c'est premièrement cette voix-là qui nous attache, nous retient et justifie notre dilection.

Je prendrai argument, pour illustrer ce qui m'a toujours paru une évidence, des romans de Tor Age Bringsværd (écrivain norvégien né en 1939) intitulés Gobi,qui furent, dès l'origine, conçus comme une œuvre à reprises (elle en est aujourd'hui à son volume V) et qui, d'une part ont obtenu dans le Nord un éclatant succès, d'autre part illustrent parfaitement les vues que je soumets ici au lecteur. Je ne m'intéresserai qu'au premier tome de la série, sous-titré La lune de l'enfance,que j'ai traduit ainsi que le volume II sous-titré Gengis Khan ${ }^{1}$.Mais en me concentrant sur le tout premier livre, qui fut une manière de " percée " personnelle de Bringsværd et où il s'est efforcé moins qu'ensuite de dissimuler ses clefs, je pense parvenir plus près de sa réalité, bien que le volume II ait été, à ce jour, le mieux accueilli en Norvège.

Le thème central de cette série - en précisant tout de suite que Bringsværd s'est intéressé à bien d'autres sujets et que c'est un auteur très prolifique, notamment en matière de parodie de la religion nordique ancienne - revient à une méditation sur le désert de Gobi et surtout sur la fantastique épopée de Gengis Khan (XII-XIII ${ }^{\mathrm{e}}$ siècle) dont nous savons le fracassant succès qu'elle connaît en ce moment partout en Occident. Voici donc les cinq volumes parus à ce jour et qui, tous, ont en sur-titre Gobi(avec, en premier sous-titre: "L'un de plusieurs livres»), le second sous-titre, qui donne sa coloration propre à chaque volume, étant :

7 1) La lune de l'enfance, 1985, Barndommens måne

2) Gengis Khan, 1987

3) La peau et les os du diable, 1989, Djevelens skinn og ben

4) Mon prince 1994, Min Prins

5) Baghdad 1997

8 Je précise que ces ouvrages ont obtenu toutes sortes de prix littéraires en leur pays (mais pas le Prix du Conseil Nordique pour lequel, pourtant, la série a été proposée officiellement à deux reprises) et que si le lecteur était intéressé par l'étude de la possible genèse de ces ouvrages, l'auteur a consacré de nombreuses pages, en fin de chaque volume, et en tout petits caractères, à la mention d'une bibliographie qui prouverait qu'il est allé se documenter aux bonnes sources, à des indications de type «technique » pour montrer qu'il n'est pas ignorant des composantes factuelles de son sujet et à de longs développements sur les circonstances précises de ses recherches et de la composition de son œuvre. Ainsi, il pourrait sembler nous fournir toutes les garanties requises à l'appréciation d'un bon roman de type historique. Tout en tirant son épingle du jeu puisque ces pages entendent certainement introduire une distance entre cet auteur et son sujet. Et nous donner à comprendre que le parti-pris de départ relève réellement de la (re)composition. Comme c'est là tout mon propos, on comprendra que j'aie été particulièrement sensible à cette attitude, surtout en matière de roman prétendument historique.

9 Ce qui m'a immédiatement frappé - et l'on admettra que ce soit là un réflexe banal de traducteur qui se doit de tenir un fil à suivre s'il veut que sa traduction présente une 
unité recevable -, c'est l'extrême diversité des thèmes abordés. Il est tout à fait clair que Gengis Khan, en tant que personnage historique, n'aura été qu'un prétexte (prétexte si l'on est lacanien) à l'entreprise de création de Bringsværd. Celui-ci a saisi une occasion qui lui a paru vraiment propice pour donner libre cours, comme je m'en suis déjà ouvert, à sa passion de conter, de raconter. Le conquérant mongol n'aura été, dans ce premier volume, que le fil directeur vaguement suivi. Or il est manifeste que Bringsværd, cet amateur d'antiquités et/ou de Moyen Age passablement exotique, avait été fasciné, depuis bien avant 1985, par toutes sortes de récits, légendes, contes, anecdotes plus ou moins étranges mais qui pouvaient, tous, fort bien avoir un soubassement d'ordre historique. Je ne prendrai que quelques exemples : a réellement eu lieu, au XII ${ }^{\mathrm{e}}$ siècle, une croisade des enfants qui a lamentablement échoué en raison de la cupidité et des malversations opérées en l'occurrence par les organisateurs de la chose. Mais voilà un thème extraordinaire qui ne pouvait pas ne pas attirer l'attention d'un bon romancier en mal d'affabulation! Ou encore: tout le monde connaît la légende du fameux preneur de rats de Hameln qui, dépité de n'avoir pas été payé selon les conventions passées par les échevins de la ville de Hameln qu'il venait de délivrer d'une invasion de rats, s'est vengé en emmenant pareillement les jeunes enfants de la ville et en les faisant disparaître. Et je n'ai pas besoin d'insister sur le formidable prestige qu'exercent, depuis huit bons siècles, Karakoroum, le désert de Gobi, la sagesse orientale, et la Horde d'Or qui parvint jusqu'à Vienne au moins !

Donc, une masse de matière narrative puisée à toutes sortes de sources et qu'il conviendrait d'organiser autour d'un axe central appelé Gengis Khan (ou le désert de Gobi dont il ne doit pas être indifférent que l'auteur l'ait privilégié, se réservant d'introduire le conquérant prestigieux en sous-titre seulement): une fantastique collection d'images (nous sommes dans les deux dernières décennies du $\mathrm{xx}^{\mathrm{e}}$ siècle, il ne faut jamais plus omettre de prendre en considération l'influence patente que le cinéma exerce sur les inspirations romanesques à caractère historique) avec une complaisance visible sur les aspects mystérieux, " policiers ", d'une affaire qui n'a toujours pas trouvé d'élucidation réellement satisfaisante.

11 Cela donne un texte curieux, "à découpage » selon les précisions qui vont suivre.

12 Je veux dire que l'auteur a entendu mener, non pas exactement de front, mais à tour de rôle, avec accent plus ou moins fort porté sur tel ou tel élément, et en se réservant le droit de revenir dessus autant de fois qu'il le juge nécessaire, une série de grands thèmes qu'il aime visiblement. Je les affecte d'un numéro de 1 à 6 . Je les appelle centraux parce qu'ils sont indispensables à la progression interne de l'action. Si on en supprime un, une manière de déséquilibre s'introduit dans l'ensemble, qui donne une narration boiteuse en quelque sorte. Voici leur nomenclature, en précisant, car la chose est d'importance, que mes découpages ne sont pas arbitraires, ils répondent à un jeu très subtil mais visiblement conscient de blancs, doubles ou triples interlignes, ou d'astérisques ou autres signes convenus qui morcellent le cours du récit :

13 1) Le personnage principal, qui fut un jeune garçon de Hameln et dit se prénommer Wolfgang, qui a pris part à la croisade des enfants, qui est maintenant plus ou moins prisonnier à Karakorum dans le domaine de Gengis Khan, sous la férule d'un grand sage appelé Ye-Liu-Chutsai, et qui serait poursuivi par d'invisibles ennemis tandis que nous l'apprendrons peu à peu - il est surveillé par d'énigmatiques espions aux responsabilités étendues, confie à un rouleau de soie (car il sait écrire) le récit de sa vie. Il médite, ce faisant, sur sa propre histoire qui a voulu qu'il fût longtemps jongleur ou 
bouffon, sur ses amitiés d'enfance (un certain Mika), ses amours, etc. Ce thème 1 concerne donc directement Wolfgang.

2) Ici, et presque dès le début du livre, nous avons à faire à Gengis Khan, plutôt à travers sa légende ou son environnement que pour lui-même (ce sera le thème du volume II dont je ne m'occupe pas ici). L'approche n'est pas directe, au contraire, elle se fait par éclairages de biais qui contribuent fortement à mettre en relief sa légende. La volonté est très nette, chez notre auteur, de dédoubler, magnifier, diluer un soubassement historique acceptable en pratiquant, comme par système, toutes sortes d'ouvertures ou glissements vers la légende, le mythe, voire la religion ou, en tout cas, une philosophie qui se voudrait orientale.

3) Ce thème est important car il justifie l'ensemble de l'ouvrage. On pourrait l'appeler Karakoroum tout court, ou bien Karakoroum et ce qui s'y passe, ouvertement et en secret, notamment autour du Lac de la Demi-Lune qui constitue une sorte de point géographique central (et dont le nom a pu suggérer le sous-titre du volume, bien que le prétexte apparent soit un poème d'un certain Li Po/ VIII siècle / et précisément intitulé «La lune de l'enfance »).

4) Pourrait rentrer dans 1, supra,mais il est tellement important, et si souvent abordé que je le mets à part: il revient aux mille et une allusions incitant à convaincre le lecteur que Wolfgang est bien espionné, poursuivi, que sa vie n'est pas du tout en sécurité. Qui sont ces espions, pour quelles autorités travaillent-ils, quelles fins poursuivraient les gens qui en veulent à la vie du héros, bien entendu, nous ne le savons pas. Cet élément est réellement moteur: il a pour visible fin d'exciter la curiosité du lecteur et de tenir l'ensemble de la narration en vie.

5) Il s'agit ici du thème, majeur en vérité en raison de ses retentissements et historiques et moraux, du preneur de rats de Hameln, directement couplé à celui de la croisade des enfants puisque, c'est là que joue à plein l'imagination de Bringsværd, les enfants en question sont les mêmes, en grande partie, que ceux qui ont été ravis à Hameln par le preneur de rats.

6) Et justement, un thème en soi s'applique au personnage de moine appelé Nicolas mais il peut tout aussi bien s'agir du fameux preneur de rats - qui à la fois ravit et effraie les enfants car il est aussi bien leur guide que celui qui les pervertira de maintes façons, notamment en Egypte.

14 A partir de ces grands motifs qui, visiblement, sont les axes majeurs de la narration, l'auteur introduit fréquemment ce qu'il faut appeler des digressions - j'entends par là des développements qui n'interviennent qu'une seule fois - mais qui jouent souvent un rôle actif dans la progression de l'action comme dans le développement de la réflexion. Soit :

7) Le récit passablement baroque et de valeur inégale concernant « les zizis » du Diable.

8) La mention de rêves ou de cauchemars dont la fonction est notoirement de faire progresser l'action par anticipations, récapitulations ou redoublements.

9) L'image des Rois Mages et de leurs fabuleux trésors.

10) Inévitablement, dirai-je, la mention de la Nef des Fous et de son sort mystérieux.

11) Trois histoires différentes de Mort destinées à intensifier une atmosphère déjà passablement morbide.

12) La présentation détaillée du roi des rats.

13) La mention de la tour aux souris de Bingen et de la légende qui s'y attache. 
14) Un conte kabyle expressément donné pour tel.

15 et 16) Deux contes chinois différents.

Cette division, dont j'ai déjà noté qu'elle n'avait rien d'arbitraire puisqu'elle est justifiée en typographie, courrait risque de dispersion ou de bric-à-brac si le récit, impeccablement dominé comme il l'est, n'avait vu l'écueil et n'y avait veillé en proposant une sorte de tissu conjonctif sous forme d'une image (ou une série d'images apparentées) encore : celle du rat, des rats ou des souris, du roi des rats, du nœud de queues de rats, la valeur symbolique de cet animal n'appelant pas d'élucidation supplémentaire. La polysémie de ce symbole, toutefois, ne doit pas être rapidement escamotée. Invasion de rats, invasion de Mongols; peste maléfique transmise par les rats, infection morale et religieuse due aux fausses religions; trahisons des clercs véhiculée par les "rats de bibliothèque », on n'en finirait pas de définir les échos que peut susciter cette figuration.

N'importe. Je voudrais simplement faire valoir la science très élaborée de la composition de ce livre, de sa structure si l'on veut.

Le livre est divisé en 12 sections bien marquées par autant de renvois à la page. Ces sections sont de longueurs à peu près égales, sauf la section $n^{\circ} 4$ (jusqu'à laquelle, inclusivement, j'irai dans ce qui va suivre) qui est considérablement plus longue parce qu'elle est la plus riche de matière, et la toute dernière section, de conclusion, qui ne couvre que trois pages.

Voici, en fonction des numéros qui ont été affectés, plus haut, aux thèmes majeurs ou secondaires, comment se présentent les quatre premières sections :

20 A) $1-2$ (ensemble) / 3/ 4/3/1/3/4/1/5

B) $1-5$ (ensemble) $/ 3 / 5 / 1 / 4 / 3 / 1$

C) $1 / 3+2$ (ensemble)

D) 5/6/5/7/5/6/1/3/1/4/1/4/8/1/5/6/2/6/5/1/3/1/4/9/1/5/1

21 Arrêtons-nous là : il ne s'agit pas de proposer au lecteur je ne sais quelle grille ésotérique, mais simplement de lui faire prendre conscience de l'étrange jeu auquel se livre l'auteur qui pourrait, donc, passer pour avoir adopté une démarche sautillante et instable, puisqu'il semble ne pas pouvoir se fixer plus de deux ou trois pages sur un motif donné. Évidemment, il y a un «je » qui s'exprime constamment, c'est Wolfgang devenu jongleur puis mémorialiste à Karakoroum, et cela seul peut servir d'agent de liaison. Mais il me parait clair que Bringsværd a voulu faire consciemment un travail extrêmement subtil où diverses incitations narratives ont tendu à constituer un tout multiface. En fait, rien n'est gratuit dans cette savante décomposition-recomposition : non seulement une sorte d'image "globale » finit par s'imposer où les enfants de la croisade, ceux de Hameln, les esclaves à Alexandrie, les cavaliers de la Horde d'Or et les sages de Karakoroum constituent une vaste symphonie, mais encore une large fresque s'établit, qui finira par constituer l'arrière-plan sur lequel s'enlèvera la prestigieuse aventure de Gengis Khan. En somme, et en un sens, il est permis de penser que Bringsværd s'est attaché, consciemment semble-t-il, à reconstituer une époque, un regard, un contexte historique qui permettent de mieux appréhender l'extraordinaire événement. Admettons, la chose est devenue quasi banale ces dernière décennies, que l'Histoire "objective", en soi, n'existe pas, que seul ait du sens le regard si possible documenté que nous jetons sur la conjoncture envisagée, que ce n'est pas l'Histoire qui est,mais notre regard, donc, pour nous en tenir à notre sujet précis, qu'il n'y pas de Gengis Khan ni de Gobi, mais notre, mon Gengis Khan, notre, mon Gobi : ce travail 
auquel se livre l'auteur va bien dans le sens de cette possible substitution à l'impossible et souvent dérisoire prétention à l'objectivité (à la " réalité »), de notre vérité présente et située : ce qui explique que l'Histoire doive être récrite à intervalles plus ou moins réguliers, et que les histoires, au pluriel et avec minuscule, vaillent peut-être mieux que l'Histoire, au singulier et avec majuscule?

On répondra, mais l'objection s'inscrit, en fait, exactement dans le fil de la démarche que je suis présentement, que nous avons affaire ici au déconstructivisme/ reconstructivisme cher à Derrida et que l'exemple de Bringsværd témoigne, une fois de plus, de l'attention extrême que l'on porte, dans le Nord, à tout ce qui se fait de nouveau en matière artistique ou littéraire dans le reste du monde. Et d'invoquer des exemples tout à fait similaires comme celui de Per Odensten le Suédois, avec Gheel, la ville des fous(1981), ou de Per Hultberg le Danois dans Requiem(1985), ou de Thor Vilhjalmsson l'Islandais écrivant La mousse grise brûle ${ }^{2}(1986)$ - sans parler, pour ne donner qu'une illustration française, de Jean d'Ormesson rédigeant son Histoire du Juif errant.Tous ces ouvrages, et bien d'autres, dans le Nord ou ailleurs, relèveraient $d u$ même type d'analyse. Et, sans aucun doute, témoignent du même regard sur le réel. A des littératures qui ont commencé par les sagas islandaises (XIII ${ }^{\mathrm{e}}$ siècle) et qui, donc, sont dès les origines totalement intoxiquées d'Histoire (d'histoires ?), cette aperception moderne de l'événement dit passé ne peut paraitre révolutionnaire.

Car, et c'est là que la question me paraît vraiment passionnante, le réflexe - si j'ose dire - de Bringsværd non seulement n'est pas nouveau, non seulement n'a pas besoin de Derrida et aliapour se justifier, mais encore trouve des modèles Scandinaves (islandais en fait) dès le XII ${ }^{\mathrm{e}}$ ou XIII ${ }^{\mathrm{e}}$ siècle.

Il s'agit des sagas islandaises, déjà évoquées, qui, elles aussi, prétendent faire de l'Histoire, elles aussi, ne résistent à peu près jamais au pur plaisir de vous raconter une belle et bonne histoire, et ne peuvent en aucun cas passer, elles non plus, pour innocentes ou gratuites. Je ne reprendrai pas ici la longue démonstration ${ }^{3}$ qui fait que, partie de la théorie dite de la Freiprosaqui aurait voulu, Romantisme oblige, que les sagas fussent spontanément nées de l'inspiration populaire et eussent livré une fidèle relation historique dûment transmise par tradition orale, la recherche a fini par adopter le point de vue radicalement opposé en voyant bien que les sagas sont des œuvres littéraires, d'auteurs parfaitement conscients des buts visés et obéissant à toutes sortes de théories - ce qui interdit de les prendre pour des documents historiques recevables ${ }^{4}$. C'est la théorie dite de la Buchprosapour laquelle j'aurai beaucoup milité depuis trente ans !

Or il se trouve que l'un des arguments majeurs qui m'ont comme obligé à renoncer à toute théorie de type populaire-spontané, tient précisément à une étude très attentive de la composition des grandes íslendingasögur.Là non plus, je ne vais pas ennuyer le lecteur par une reprise de démonstrations en forme que j'ai faites il y a maintenant plus de deux décennies. Mais si l'on regarde de près un superbe texte comme Eyrbyggja saga (La Saga de Snorri le godi)ou la non moins réussie, bien que relevant d'une autre catégorie (konungasagaou saga royale), Saga de Haraldr VImpitoyable (Haralds saga Sigurdarsonar,dans la Heimskringlade Snorri Sturluson) $)^{5}$, on aboutit à la conclusion comme inéluctable que chacun de ces deux textes est l'œuvre d'un écrivain de haut vol 
(inconnu dans le premier cas, tout à fait célèbre dans le second) parfaitement maitre de ses techniques, ayant organisé avec le plus grand soin sa matière en fonction d'effets très précis à créer, bref, le contraire exact d'un improvisateur. Le premier (Eyrbyggja)travaille par thèmes et sous-thèmes, le thème $A$ appelant un thème $B$ avec, dans l'intervalle, élaboration plus ou moins rapide ou épisodique d'un sous-thème $A$ (lequel peut fort bien, à son tour, engendrer un motif $\mathrm{N}$, etc.) selon un schéma idéal du type : vérité, en soi. La réflexion qui, malheureusement, ne s'est guère exercée sur des thèmes proprement historiques, d'un Per Olof Sundman, serait la bienvenue ici -, je suis en ce 
moment au Moyen Age et parle des sagas islandaises que je vois comme des textes infiniment travaillés. Est-ce uniquement à des fins artistiques? Non, je ne le pense pas. Les sagnamenn(auteurs de sagas) étaient à peu près tous des clercs ou des hommes ayant reçu une formation tout à fait cléricale, on ne le niera pas. Et donc, ce qu'ils écrivaient n'était pas de l'Histoire selon notre acception du terme, c'était de l'historia: comprenons un récit qui n'existe pas en soi, qui se doit à toute fin d'avoir une raison d'être, laquelle est exemplaire, ne peut pas ne pas l'être. Il n'y a pas de littérature profane à ces époques-là. Le lecteur doit toujours pouvoir (et l'auteur doit toujours donner matière à) " attraire à moralité », comme on disait alors. Il s'ensuit que le motif doit être arrangé, remodelé, "repensé» en fonction de l'objectif visé et que dans ces conditions, il devient un peu dérisoire de vouloir à tout prix parler de vérité historique ou de fidélité à la réalité.

Suis-je en train de suggérer que l'art de Tor Åge Bringsværd rejoindrait celui de Snorri Sturluson, entre autres? Oui. Ce sont historiens dans la mesure où ils entendent nous restituer leur propre image (leur ou celle que leur ont inculquée leurs divers maîtres, quels que soient leurs noms, mais la distinction n'est-elle pas spécieuse ?) d'un donné dont - cela, nous le savons, aujourd'hui - personne, jamais ne pourra avoir la prétention d'affirmer l'identité avec le modèle « réel ».

Et d'ailleurs, lisez d'un peu plus près l'auteur de Gobi: je me suis proposé, dit-il, d'écrire ce livre : "Je contemplerai les images que j'ai dans la tête», (op.cit., p. 12). Et encore : histoires ou Histoire? Voici : "J'aime les histoires de faits merveilleux. Mais cela ne signifie pas que je croie tout ce que je vois et entends » (ibid.,p. 95) - avec cette manière de confession désespérée : «Il y a tant d'histoires que j'aimerais bien raconter. Et pas seulement les miennes propres...»(ibid.,p. 99), et cette sorte de cri du cœur qui pourrait bien servir d'exergue à mes modestes propos présents : il veut, nous dit-il, nous rapporter une «belle histoire. Si belle qu'il faut lui permettre de vivre. Car cette nuit, je veux y croire...» (ibid.,p. 108).

Et puis... je suis parti de la passion de raconter qui m'a toujours paru posséder, au sens fort du verbe, un bon écrivain Scandinave. Histoire, histoire(s) : elles reviennent toutes à raconter, n'est-ce pas? Or relisons une dernière fois Tor Åge Bringsværd : « Mais je m'éloigne de ce que je voulais écrire. Je ne parviens pas à repousser mes pensées. Je vagabonde. Je raconte comme un jongleur qui court après une balle : chaque fois qu'il se penche pour l'attraper, il lui donne involontairement un nouveau coup de pied. Et chaque fois, il est tout aussi surpris. Voilà comme il court... encore et encore... dans une interminable quête de l'impossible. » (ibid.,pp. 169-170).

C'est peut-être cela, cet impossible fascinant, qui est l'Histoire, qui suscite les histoires, que prétendait déjà cerner l'historia... 


\section{NOTES}

1. Pour le volume I chez Arcane 17, Saint-Nazaire, 1991, pour le volume II, chez Joseph K, Nantes, 1995.

2. Le roman de Per Odensten a été traduit en français (Arcane 17) en 1991, celui de Thor Vilhjamsson (Actes Sud) également. Celui de Per Hultberg attend toujours un traducteur.

3. Elle existe, en grand détail, dans Régis Boyer : Les sagas islandaises, Paris, Payot, 1978, $3^{\mathrm{e} e ́ d .}$ 1992, chap. 3 et 4 .

4. Bilan de la question dans Régis Boyer: «Les sagas islandaises sont-elles des documents historiques?» dans Recueil d'études en hommage à Lucien Musset. Cahiers des Annales de Normandie n²3, Caen, 1990, pp. 109-126.

5. L'une et l'autre existent en français : la Saga de Snorri le godi chez Aubier, 1973 (reprise dans

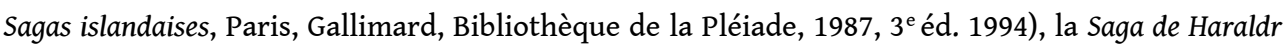
l'Impitoyable chez Payot, Payot-Poche, 1979. Les renvois iraient aux pages où l'analyse de la composition de l'une et de l'autre a été faite, avec schémas à l'appui, soit, pour Eyrbyggja saga, pp. 37 ssqq. ; pour Haralds saga, pp. 26 et sq.

\section{RÉSUMÉS}

L'auteur part d'une double constatation: le genre narratif, notamment roman ou surtout nouvelle, a toujours, semble-t-il, été une sorte de spécialité Scandinave ; la passion de conter, de raconter paraît faire partie des composantes inaliénables du génie nordique. En sorte que prétendre faire de l'Histoire, avec majuscule, désir dont nous savons aujourd'hui la vanité, revient ici avec constance à vouloir raconter des histoires.

Cette opinion est étayée par une analyse rapide de Gobi, la lune de l'enfance, du romancier norvégien actuel Tor Åge Bringsværd, ce livre, premier d'une série parvenue aujourd'hui à son cinquième volume, étant finalement une méditation autour de l'extraordinaire destinée de Gengis Khan. Il n'est pas difficile de montrer que ce roman est composé autour d'une demidouzaine de thèmes principaux, tous fondés sur des légendes ou réminiscences plus ou moins historiques, qui engendrent volontiers des sous-thèmes provisoires, thèmes et sous-thèmes apparaissant, reparaissant, interférant selon une technique consciente et fort élaborée, un peu comme un travail hautement artisanal de marqueterie ou de mosaïque ou de tapisserie. C'est le lieu de faire remarquer que les Scandinaves, depuis que nous les connaissons, ont toujours été, dans tous les domaines, d'ornementateurs, de décorateurs, des artisans au plus noble sens du terme, ardents à pousser les possibilités d'expression du matériau qu'ils travaillent à une sorte de point de perfection.

Or cette conception s'applique parfaitement aux sagas islandaises (XIII ${ }^{\mathrm{e}}$ siècle), par lesquelles la « littérature » a débuté, avec un éclat nonpareil, dans le Nord. La continuité, ici saisie à travers l'œuvre d'un écrivain donné (mais il serait tout à fait facile de prodiguer d'autres exemples) est patente. Or les sagas islandaises, œuvres de clercs, entendaient " attraire à moralité ", c'est-àdire que le genre de l'historia (évoquer le passé pour en tirer une leçon) dictait et l'éventuel souci 
d'écrire de l'Histoire, et la passion de narrer des histoires. Il semble que ce soit sous cet angle que le roman dit historique puisse être envisagé avec le plus de fruit dans le Nord...

Der Autor geht von einer doppelten Feststellung aus: Die erzählende Gattung, vor allem der Roman und die Novelle, war immer, so scheint es, eine skandinavische Spezialität. Die Leidenschaft zu berichten, zu erzählen, ist offensichtlich Teil des unverwechselbaren Genies der nordischen Länder, so daß Geschichte machen zu wollen, ein Wunsch, von dem wir heute wissen, wie nichtig er ist, hier ständig als Wunsch, Geschichten zu erzählen, wiederkehrt.

Diese Meinung stützt sich auf eine kurze Analyse von Gobi, der Mond der Kindheit, des zeitgenössischen norwegischen Romanciers Tor Åge Bringsgærd. Dieses Buch, das erste einer Reihe, die heute schon aus fünf Bänden besteht, ist schließlich eine Betrachtung über das außergewöhnliche Schicksal von Dschingis-Khan. Es ist nicht schwer aufzuzeigen, daß dieser Roman um ein halbes Dutzend Hauptthemen angelegt ist, die alle mehr oder weniger auf historischen Legenden oder Erinnerungen basieren und die absichtlich vorläufige Unterthemen hervorbringen: Themen und Unterthemen erscheinen und überlagern sich nach einer bewußten und gut ausgearbeiteten Technik, ein bißchen wie eine kunsthandwerkliche Intarsien- Mosaikoder Tapisseriearbeit. Es muß hier gesagt werden, daß die Skandinavier, solange wir sie kennen, immer und in allen Bereichen Verzierer, Dekorateure, also Handwerker im besten Sinne des Wortes waren, mit dem sehnlichen Wunsch, die Ausdrucksmöglichkeiten des Materials, das sie bearbeiten, zur Perfektion zu bringen.

Nun ist diese Konzeption sehr gut auf die Islandische Saga (13. Jahrhundert) anzuwenden, die die « Literatur » in den nordischen Ländern sofort zu einer Blüte ohnegleichen geführt hat. Die Kontinuität, hier aufgezeigt am Werk eines bestimmten Autors (doch es wäre nicht schwer, das auf andere Beispiele auszudehnen) ist offenkundig. Nun beabsichtigen die isländischen Sagas, Werke von Klerikern, « zur Sittlichkeit hin zu erziehen », das heißt, daß die Gattung der historia (die Vergangenheit heraufbeschwören, um daraus eine Lehre zu ziehen) sowohl das mögliche Bemühen, Geschichte zu schreiben, als auch die Leidenschaft, Geschichten zu erzählen, bestimmte. Es scheint, daß der sogenannte historische Roman in Nordeuropa unter diesem Aspekt am fruchtbarsten betrachtet werden kann...

\section{AUTEUR}

\section{RÉGIS BOYER}

Université de Paris IV - Sorbonne 